\title{
Hypermutation as a Factor Contributing to the Acquisition of Antimicrobial Resistance
}

\author{
Jesús Blázquez \\ Departamento de Biotecnologia Microbiana, Centro Nacional de Biotecnologia, Consejo Superior de Investigaciones Cientificas, Campus Universidad Autonoma \\ de Madrid, Spain
}

Contrary to what was thought previously, bacteria seem to be, not merely spectators to their own evolution, but, through a variety of mechanisms, able to increase the rate at which mutations occur and, consequently, to increase their chances of becoming resistant to antibiotics. Laboratory studies and mathematical models suggest that, under stressful conditions, such as antibiotic challenge, selective pressure favors mutator strains of bacteria over nonmutator strains. These hypermutable strains have been found in natural bacterial populations at higher frequencies than expected. The presence of mutator strains in the clinical setting may indicate an enhanced risk of acquiring antibiotic resistance through mutational and recombinational events. In addition, some antibiotics are inducers of mechanisms that transiently increase the mutation rate, and thus probably act, not only as mere selectors of antibiotic resistant clones, but also as resistance-promoters.

Societies_-at least the developed ones_are facing the problem of an increasing number of antibiotic-resistant microbial pathogens. Paradoxically, this is a consequence of the success of antibiotic therapy. This success led to the belief that any infectious disease could be treated, and even eliminated, with these "miraculous" drugs. Nevertheless, during the past 6 decades, we have been witness to one of the most rapid and striking phenomena of biological evolution, which has been provoked, unfortunately, by humankind: the adaptation of bacteria to antibiotics. The extended use-and, all too often, abuse and misuse-of these molecules has produced an impressive effect, leading to the selection and spread of resistant bacteria.

Bacteria may acquire antibiotic resistance in 2 main ways: through horizontal transfer (i.e., acquisition of already-made and pretested resistance genes from other microorganisms) and through mutation in different chromosomal loci. Only the latter will be considered here. Although it has been stated that, be-

Received 13 May 2003; accepted 5 July 2003; electronically published 30 September 2003. Financial support: This work was supported in part by the Ministerio de Sanidad y Consumo (Fondo de Investigación Sanitaria) (grant 01/0020-02) and the Ministerio de Ciencia y Tecnología (grant BMC2001-0012), Spain.

Reprints or correspondence: Dr. Jesús Blázquez, Departamento de Biotecnologia Microbiana, Centro Nacional de Biotecnologia, CSIC, Campus Universidad Autonoma de Madrid, Cantoblanco, 28049 Madrid, Spain (blazquez@cnb.uam.es).

Clinical Infectious Diseases 2003;37:1201-9

(C) 2003 by the Infectious Diseases Society of America. All rights reserved. $1058-4838 / 2003 / 3709-0010 \$ 15.00$ cause of the low frequency at which mutations occur, resistance in natural environments is mainly acquired through horizontal transfer [1], a number of mechanisms of antibiotic resistance are based on mutational events [2]. The sequence of a gene encoding the target of an antibacterial molecule may be altered by mutation, leading to the inability of that molecule to inhibit its activity. For instance, most of the known mechanisms of bacterial resistance to some antibiotics, such as rifamicins and fluoroquinolones, are caused by mutations in the genes encoding the targets of these molecules (RpoB and DNA-topoisomerases, respectively). Variation in the expression of antibiotic uptake or of efflux systems may also be modified by mutation, leading to an increased resistance to antibiotics. For instance, the reduced expression or absence of the OprD porin of Pseudomonas aeruginosa reduces the permeability of the cell wall to carbapenems [3]. Most of the antibiotic resistance phenotypes associated with uptake and with efflux systems are caused by mutations in regulatory genes or their promoter regions [4]. An additional problem caused by those mutations that lead to increased expression of efflux systems is that, in general, such mutations confer resistance to multiple antibiotics. For example, mutations in the Escherichia coli mar gene affect the expression of about 60 different genes, including down-regulation of $\mathrm{OmpF}$ and up-regulation of AcrAB. AcrAB is involved in the efflux of $\beta$-lactams, fluoroquinolones, chloramphenicol, and tetracycline [5]. In P. aeruginosa, mutation in 
mexR up-regulates the mexA-mexB-oprM operon and raises the MICs of most $\beta$-lactams, fluoroquinolones, tetracyclines, chloramphenicol, and macrolides [4]. Finally, overproduction of antibiotic-inactivating enzymes may be achieved through mutational events. Many gram-negative microorganisms produce chromosomal $\beta$-lactamases (such as AmpC) at low levels. Mutations producing up-regulation of their expression, and, consequently, constitutive hyperproduction of AmpC, lead to resistance to most cephalosporins [6].

In addition, there are some clinically relevant pathogens for which plasmid- or transposon-mediated mechanisms of resistance have not been reported. Mycobacterium tuberculosis, which lacks these horizontal transference mechanisms, seems to be genetically isolated in the infected patients [7-10]. Consequently, it has to acquire antibiotic resistance by mutational events exclusively. Despite this unique way of acquiring resistance to antibiotics, mutation-mediated resistance is a major therapeutic problem that has forced clinicians to use combined antibiotic regimens to avoid development of resistance. However, epidemics of untreatable, multidrug-resistant M. tuberculosis have been described $[11,12]$. Another example may be chronic infection with $P$. aeruginosa of the lungs of patients with cystic fibrosis. This bacterium, once present, is almost impossible to eradicate, mainly because of the development of resistance to multiple antibiotics. As with $M$. tuberculosis, resistance, in this particular environment, is achieved through chromosomal mutations that are able to produce resistance to all antibiotics used in clinical practice, without the need for acquisition of exogenous DNA [13]. Moreover, most bacterial infections are produced by a relatively small number of cells (a quasi-clonal population), and there is a low likelihood that such a population will contain, by chance, a resistant variant. If these cells are sensitive to the antibiotic administered and there are no antibiotic-resistant microorganisms capable sharing their resistance genes in the new environment, then there are not too many possibilities for bacteria to acquire resistancegenes by horizontal transfer during the course of the infection. In these cases, the only possibility of becoming resistant to an incoming antibiotic is through mutations.

Horizontal transfer and mutation can act in a synergistic way because horizontal transfer introduces new alleles into a population and mutation produces new variations of these alleles. This is the case for some antibiotic-resistance genes, such as $\beta$-lactamases encoding bla-TEM genes $[14,15]$. TEM- 1 and TEM-2, which mainly confer resistance to penicillins, were disseminated by horizontal transfer through plasmids and transposons among different bacterial genera. To respond to the use of new cephalosporins, bacteria produced new TEM derivatives containing mutations that enabled them to hydrolyze these new compounds [16]. To fight this bacterial antibiotic-resistance mechanism, third-generation cephalosporins (resistant to hy- drolysis by these $\beta$-lactamases) and suicide inhibitors (such as clavulanate and tazobactam, which are able to restore the activity of penicillin against the $\beta$-lactamase producers) were developed. Unfortunately, within a few years after the development of these drugs, there were dozens of new TEM derivatives. These derivatives arose through the acquisition of mutations conferring resistance to $\beta$-lactamase inhibitors [17] or to thirdgeneration cephalosporins. These new TEM derivatives are now being disseminated by horizontal transfer through plasmids and transposons [6].

Thus, bacteria confronted with the previously described stressful situations may benefit from an increased rate of mutation (i.e., the production of a higher number of mutations per cell per generation).

Mutation, mutation rate, and stable hypermutation. The word "mutation," according to many textbooks, refers to any permanent, and consequently heritable, change in the DNA sequence. The word "mutant" refers to an organism that is the direct offspring of a normal member of the species (the wild type), but that differs from a normal member of the species in that it has acquired a mutation. The "mutation rate" can be simply defined as the chance of mutation to a particular phenotype; for instance, the chance of developing resistance to an antibiotic.

Only 60 years ago, microbiologists believed that bacteria adapted to environmental stress by a process of directed change (i.e., Lamarckian inheritance), rather than as the result of random mutations and natural selection (i.e., neo-Darwinian theory). This belief was based on the observation that bacteria exposed to a toxic substance seemed to become resistant in response to that substance. Luria and Delbrück [18], Newcombe [19], and, some years later, Lederberg and Lederberg [20] published the results that, elegantly, buried the directedchange hypothesis for antibiotic resistance. They definitively demonstrated that bacteria acquired antibiotic resistance by mutation, independently of their exposure to the antibiotic, and that the antibiotic merely acts as a selector of the preexisting resistant variants that, finally, become fixed in the population.

It has been widely accepted for a long time that mutation is the unavoidable consequence of errors produced in the DNA replication process and/or of the failure of the error-avoidance systems. Maintaining the stability of genetic information is vital for the perpetuation of species. Thus, DNA replication must be almost free of error. For this reason, evolution has resulted in the development of a DNA replication apparatus that minimizes mistakes and of a series of systems that protect and repair DNA. However, evolution, which occurs through genetic variation and selection, is the fundamental strategy of life, allowing organisms to adapt to new environments or to adverse conditions. Without mutation, there are no new genes, no new alleles, and, finally, no evolution. Mutation is the ultimate 
source of the heritable variation on which natural selection acts. It seems that nature selected those organisms that possess a mutation rate that compromises between adaptability and adaptedness [21]. Because most of the newly arising mutations are neutral or deleterious (whereas only rare, specific mutations, such as those conferring antibiotic resistance, are favorable), it has been argued that the mutation rate has evolved to be as low as possible [22-24]. Despite this theory, clones with an elevated mutation rate (hypermutable strains, or mutators) can increase in frequency among laboratory bacterial populations [25-27]. More recently, computer simulations have suggested that mutator alleles can play an important role in adaptive evolution. Because these alleles increase the possibility of favorable mutations, they can accelerate the evolutionary rate under some conditions. During this process, mutators can be fixed in the population by "hitchhiking" with the favorable mutations they have originated [28]. Thus, the acquisition of a mutator phenotype will increase the chances of acquiring antibiotic resistance by mutational events.

There have been descriptions of mechanisms that, by increasing the mutation rate, may facilitate the adaptation to different stimuli in bacterial populations. These include the presence of inducible or transient mutators [21], hypermutable genetic sequences [29], and naturally-occurring stable mutators $[13,30-32]$. As many as $1 \%$ of the E. coli and Salmonella natural isolates are already stable strong-mutators (i.e., those with a heritable, very high mutation rate) $[30,31]$, and $20 \%$ of Pseudomonas aeruginosa and 14\% Staphylococcus aureus strains isolated from the lungs of patients with cystic fibrosis are also strong mutators $[13,33]$. The heritable hypermutation in $E$. coli, S. typhimurium, Neisseria meningitidis, and P. aeruginosa is mainly produced by alterations in the methyl-directed mismatch repair (MMR) system [13, 30-32, 34, 35]. The genes affected in the studied hypermutable strains are, in order of decreasing frequency, mutS, mutL, $m u t H$, and $m u t U(u v r D)$ [35], although deficiency in other genes can also lead to the mutator phenotype [36]. To initiate repair of a mismatch, MutS protein forms a homodimeric complex that binds mismatches. The MutS-mispair complex recruits a MutL homodimer that is required to activate $\mathrm{MutH}$ endonuclease. This endonuclease incises the newly replicated DNA strand (recognized because it is unmethylated) at 5'-GATC hemimethylated sites. The nicked strand is then unwound by helicase II (UvrD) and degraded by the action of exonucleases. Finally, repair synthesis fills in the gap [37]. Thus, a bacterial strain unable to perform any of these activities will be unable to repair mismatches and, consequently, its mutation rate will increase.

Mutators and recombination. Deficiency of either MutS, MutL, or MutH increases, not only the mutation frequency, but also the rate at which "homeologous" recombination occurs [38]. That is, it increases the rate at which 2 divergent sequences recombine. Thus, the probability of acquiring new functions, by both mutation and recombination, is greatly increased in MMR-deficient strains.

Recombination in vivo requires nearly-perfect homology between the 2 complementary DNA strands; it is almost completely abolished in $E$. coli when sequence divergence is on the order of $16 \%-20 \%$. The requirement for DNA sequence homology is greatly relaxed in MMR-deficient mutants [39], because MutS and MutL proteins presumably recognize noncomplementary nucleotides in the heteroduplex DNA intermediates, preventing recombination. For instance, interspecies recombination between $E$. coli and S. typhimurium (the 2 of which are $\sim 16 \%$ divergent in DNA sequence) is increased by up to 1000 -fold in mutS, mutL, or mutH-deficient mutants. Computer simulations suggest that MMR deficiency can accelerate bacterial adaptation if genetic variability is present in DNA fragments that have $\leqslant 30 \%$ divergence [40].

Antibiotic resistance genes may be acquired by conjugation, transformation, and/or transduction and, in some cases, further incorporated into the recipient chromosome by recombination. These genes may contain single mutations or more-severe sequence changes (i.e., donor and receptor DNA sequences may be partially divergent). The perfect example of a relationship between stable hypermutation/hyperrecombination status and acquisition of antibiotic resistance would be the case of $S$. pneumoniae, in which transformation and recombination seem to be the major sources of genetic variability [41]. In S. pneumoniae, resistance to penicillin has emerged through the development of altered penicillin-binding proteins (PBPs) with decreased affinity for this $\beta$-lactam. Mosaic $p b p$ genes containing regions from other Streptococcus species have been found in resistant clinical isolates [42]. Inactivation of the S. pneumoniae MMR-homologous system (the Hex system) can increase transformation frequencies for point mutations in homologous DNA up to 20-fold [43]. However, the Hex system becomes saturated if there is $1.7 \%-10.3 \%$ divergence between donor and recipient DNAs [44]. Consequently, the acquisition of mosaic $p b p$ genes via transformation and recombination does not seem to be improved in S. pneumoniae Hex-deficient mutants, at least in this range of sequence divergence. Nevertheless, if there is a higher percentage of sequence divergence, the Hex system seems to work efficiently [44]. Thus, one can imagine that Hex-deficient $S$. pneumoniae strains may have an improved ability to recombine very divergent sequences. Also, point mutations that decrease susceptibility to cefotaxime, such as Thr242 to Ile in PBP3 and Thr-550 to Ala in PBP2x, have been described [45-47]. For these reasons, a role for Hex-deficient mutants in the acquisition, through transformation and/or recombination, of highly divergent sequences and single-point mutations conferring antibiotic resistance cannot be ruled out in natural S. pneumoniae strains. 
Chance and opportunity. Bacteria must not only be lucky enough to find the beneficial mutation that protects them from the inhibitory action of antibiotics, but they must also have the opportunity to express it. Once the resistance mutation occurs, the resistance phenotype is not immediately apparent, because enough mutant (i.e., resistant-associated) protein should be produced to protect the cell against the antibiotic or to pump the antibiotic outside of the cell before executing its inhibitory action (the so-called "phenotypic lag phenomenon"). Thus, it is important that the newly-produced resistant variants are not immediately confronted with high concentrations of the antibiotic. However, the effect of antibiotics may not be immediate, because they require some time to exert their inhibitory action. In this sense, we have seen that even high concentrations of different antibiotics allow several replication rounds of treated $P$. aeruginosa cells (A. Oliver and J. B., unpublished data). Thus, even in the presence of antibiotics, mutants that have recently appeared may express enough mutant proteins to protect the cell before the definite action of the antibiotic. These results, together with pharmacokinetic and other arguments, reinforce the idea that infections should be rapidly and aggressively treated with antibiotics (i.e., with a large dosage) to reduce the possibility of resistance acquisition.

Transient hypermutators. Even if high mutation and/or recombination rates increase the probability of adaptation in the short term, once the selective pressure disappears, as, for instance, when antibiotic resistance has been achieved, the hypermutation and/or hyperrecombination phenotype of MMR deficiency may have a tremendous evolutionary cost for bacteria because most of the mutations are neutral or deleterious $[22,23]$. Obviously, the production of mutations only when needed (transient mutation) might be less costly. This kind of strategy could "turn on" the mutator activity only under stress conditions and "turn off" the mutator activity when it was unnecessary. The SOS repair system in bacteria is the paradigm of a transient mutator system. This system, when induced by DNA damage, activates a series of $>40$ different genes that are regulated by the product of the lexA gene [48]. Among these genes are $p o l B, \operatorname{din} B$, and $u m u C D$, encoding the so-called "error-prone," or specialized, DNA polymerases II, IV, and V, respectively [49]. These enzymes are able to overcome the blockage of DNA replication produced by damaged DNA sequences, but, when operating on nonsubstrate templates or on copy noncognate damaged sequences, they exhibit reduced fidelity and, consequently, produce mutations in the newlysynthesized DNA strand (for a review, see [50]). Very recently, Boshoff et al. [51] reported that the production of DnaE2 (encoded by the dnaE2 gene), an additional copy of the major replicative DNA polymerase (DnaE) in M. tuberculosis, is induced by DNA damage. Resistance to rifampin emerged in vivo more frequently in the wild type than in the dnaE2 knockout strain. The authors conclude that DnaE2 may contribute to the emergence of drug resistance in vivo through inducible mutagenesis.

Thus, as a consequence of DNA damage, the mutation rate of bacteria will be increased. When the DNA-damaging agent disappears, the original mutation rate is recovered. It should be noted that some antibiotics used in clinical practice, such as fluoroquinolones, are good inducers of the SOS system (see below). In addition to its effect on the mutation rate, induction of the SOS system increases recombination between both homologous and divergent bacterial DNA sequences through the overproduction of RecA, which mediates recombination [38, 52]. It is of interest that it has been demonstrated that a strong SOS induction in MMR-deficient cells allows the full interchange of very divergent sequences and completely abolishes genetic barriers between E. coli and Salmonella typhimurium [53]. Thus, the combined effects of both stable and transient hypermutation and/or hyperrecombination in bacterial populations subjected to stress, such as that produced by an antibiotic, may increase the chance of acquiring antibiotic resistance, even in small populations. Also, a transient hypermutable state may be acquired through MMR depletion under stressful situations, such as the stationary phase [54].

The importance of inducible transient hypermutation in nature has been pointed out by Bjedov et al. [55]. They found that $>50 \%$ of the 787 worldwide natural strains of $E$. coli studied showed a high rate of stress-induced mutagenesis. However, it is important to note that, to be effective, the induction of transient hypermutation must occur under nonlethal conditions. In the case of antibiotics, the induction should occur using doses that, although sublethal, are close to lethal, because low concentrations of the antibiotic will not induce the transient hypermutation system, and high concentrations will kill most of the cells in the population.

Hypermutation and indirect selection for antibiotic resistance. Microorganisms harboring an antibiotic resistance mechanism acquired through either horizontal transfer or mutation will be positively selected in the presence of the antibiotic. Simply put, such microorganisms will survive, whereas those microorganisms that are sensitive to the antibiotic will be cleared from the population. After several rounds of replication, antibiotic resistant clones will become the predominant population, and, again, by chance, a new mutant that is resistant to a new antibiotic will appear in the population (figure 1A). This is the classical view of resistance acquisition and multidrug-resistance accummulation. Obviously, this is the simplest scenario, in which there is no resistance incompatibility [56] (i.e., no negative interaction between 2 different resistances), and the cost of resistance in the absence of the antibiotic is low enough to be afforded $[57,58]$. However, there is evidence 

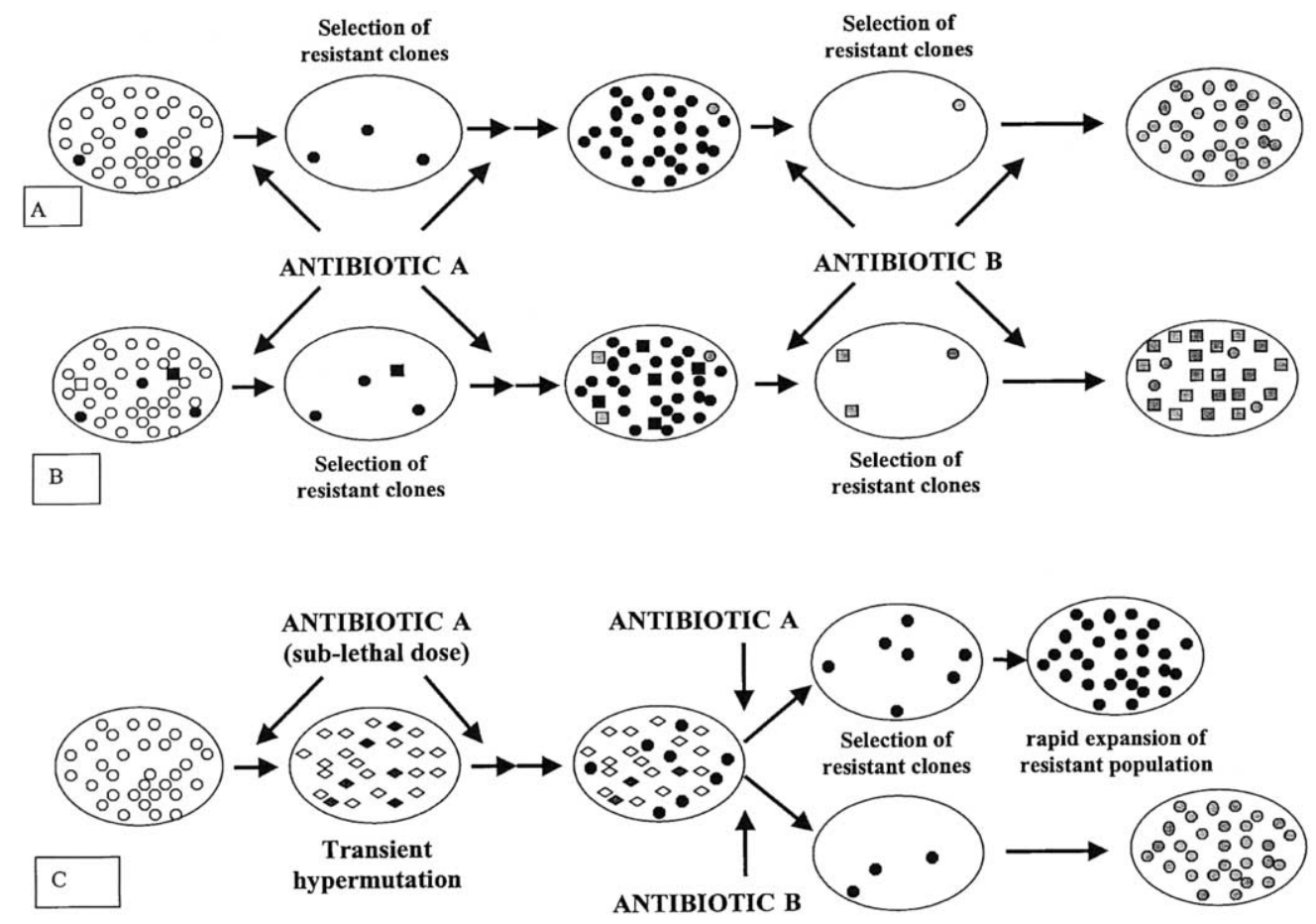

Figure 1. Simple representation of 3 of the possible scenarios, showing mutational responses of a bacterial population to consecutive antibiotic challenges. A represents the classical view: a lethal dose of antibiotic A will select the preexisting resistant clones (black circles). After a period of antibiotic pressure, only the resistant clones will grow, and the whole population will become resistant. When the population size is large enough, some clones that are resistant to the new antibiotic B (gray circles) will appear by chance. If the infection is now combated with antibiotic B, then selection of resistant clones will occur again. Note that these new clones are now resistant to both antibiotics $A$ and $B$. This continuous selection with different antibiotics may occur if bacterial populations have enough time to reach a high density and acquire, by chance, new mutations. $B$ represents the same scenario but includes the presence of mutator clones in the population. A lethal dose of antibiotic A will select the preexisting resistant clones (black circles), as in $A$, but, in this case, one of the mutator clones (squares) also harbors the mutation that confers resistance to antibiotic A (black square). Again, only resistant clones will survive, and they will grow to form an entire population of resistant clones. Note that the proportion of mutator clones has now increased [59]. Because of their higher mutation rate, mutator clones will have more chances of producing mutations that confer resistance to antibiotic B (gray squares), and, finally, they will become predominant when antibiotic B enters the scene. The net probability of acquiring successive antibiotic resistances by mutation (or homeologous recombination) is greatly enhanced because almost the whole population consists of mutator clones. $C$ represents a scenario in which a low dose of antibiotic $A$ has been administered (or the antibiotic is not able to reach, at high concentration, the site of infection). In this case, low doses of antibiotic A produce a transient mutator state, inducing, for instance, the SOS system (white diamonds). As the transient mutator state affects most cells, the probability of acquiring, by chance, a mutation conferring resistance to antibiotic $A$ increases and several clones may acquire such a mutation (black diamonds). The increased mutation rate also leads to the production of other types of mutations, including those conferring resistance to antibiotic B (gray diamonds). Although antibiotic A is administered at a low dose, clones that are resistant to it are supposed to be fitter than the clones that are sensitive to it. Thus, resistant clones may be slightly positively-selected, increasing in number during the course of an inappropriate treatment. The population is now prepared to confront higher doses of antibiotic $\mathrm{A}$ because a relatively high number of resistant mutants exist. Also, some mutants resistant to antibiotic B are present in the population. Thus, changing the antibiotic will not assure the success of the treatment. The only possibility of success is to use a combination of 2 antibiotics.

indicating, not only that antibiotics select for antibiotic-resistant strains, but also that antibiotics can act as indirect promoters of antibiotic resistance. Bacterial populations contain a subpopulation of strong mutators (i.e., cells with a $\geqslant 100$-fold increase in the mutation rate): a proportion of $\sim 10^{-5}(0.001 \%)$ [59]. The reason for this is easy to explain. In general, the proportion of a population that fortuitously contains a defective gene is $\sim 10^{-6}$. Because there are $\sim 5$ genes whose deficiency produces a strong mutator phenotype, the final proportion of mutators will be $\sim 10^{-5}$. In the same seminal paper, Mao et al.
[59] demonstrated that a single selection for a mutant phenotype increases the proportion of mutators in the selected population from the original $0.001 \%$ to as much as $0.5 \%$. Furthermore, successive selections can increase the proportion of mutator strains in the selected population to $100 \%$ [59].

This poses an interesting and remarkably practical question: are successive and prolonged antibiotic treatments increasing the possibility that bacteria will acquire new antibiotic resistances? If, as demonstrated by Mao et al. [59] in laboratory populations, single or successive selections with antibiotics in- 
crease the proportion of strong mutators in natural populations, then we can conclude that a given antibiotic not only selects for resistance to itself but can also, by increasing the proportion of mutators, indirectly select for the increased capacity to acquire resistance to other antibiotics (figure 1B). Such a selective process has been suggested by Giraud et al. [60], who found, in an intestinal colonization model, that some antibiotics select for both antibiotic resistant bacteria and mutator alleles.

It has been suggested that conditions that select for mutator strains in the clinical setting may occur in the lungs of patients with cystic fibrosis $[13,33]$. The lungs of most of these patients (as many as $80 \%$ ) are chronically colonized with P. aeruginosa, which contributes in a major way to the damage to their lungs [61]. After years of antibiotic treatment, P. aeruginosa ultimately becomes resistant to most of the antibiotics used in the treatment. We have demonstrated that as many as $20 \%$ of the $P$. aeruginosa strains isolated from these patients are strong mutators and that there is a strong linkage between the mutator phenotype and its evolution to antibiotic resistance [13]. These results strongly suggest that one of the main forces driving the selection of mutator strains, and, consequently, antibiotic resistance, may be precisely the pressure exerted by antibiotic therapy.

Antibiotics can directly increase mutation frequency. It has been found that environmental and physiological stress conditions can transiently increase the mutation rate in bacteria [55, 62]. Antibiotics, as stress producers, may, under certain circumstances, increase the mutation rate. This has been demonstrated for fluoroquinolones and aminoglycosides. Fluoroquinolones, as agents that stop DNA replication, induce the
SOS system in Salmonella and E. coli [63-65]. This induction leads, consequently, to an increase in mutation rate [65]. Streptomycin, an aminoglycoside, is known to promote mistranslation and induce a recA- and $u m u D C$-independent mutator phenotype [66]. In addition, results from our laboratory [67] indicate that $\beta$-lactams, molecules known to act as inhibitors of cell-wall synthesis but not of DNA replication or translation, also increase the mutation frequency in E. coli through the induction of the SOS system and the error-prone DNA polymerases. Although most of the resulting mutations produced by these antibiotics are expected to be deleterious, some of them may be advantageous and may lead, for instance, to the acquisition of resistance to the challenger antibiotic. In addition, the transient increase in mutation rate may lead, as in the case of stable mutators, to the production of mutations that confer resistance to unrelated antibiotics (figure 1C).

Studies on the linkage between hypermutation and antibiotic resistance. There are currently mixed opinions about the importance of hypermutability in the development of antibiotic resistance in bacteria. Nevertheless, a number of studies strongly suggest a possible association between bacteria with high mutation rates and the acquisition of antibiotic resistance. Hypermutation may be an important property of staphylococcal clones that demonstrate intermediate resistance to vancomycin. Schaaff et al. [68] found that a 4-fold higher level of vancomycin resistance could be reached in a mutS-deficient mutator strain of $S$. aureus than in the wild type strain. In addition, vancomycin resistance developed much faster in the mutator strain, suggesting that a high mutation-frequency could be one of the key factors in the emergence of intermediate-level vancomycin resistance in this species [68]. How-

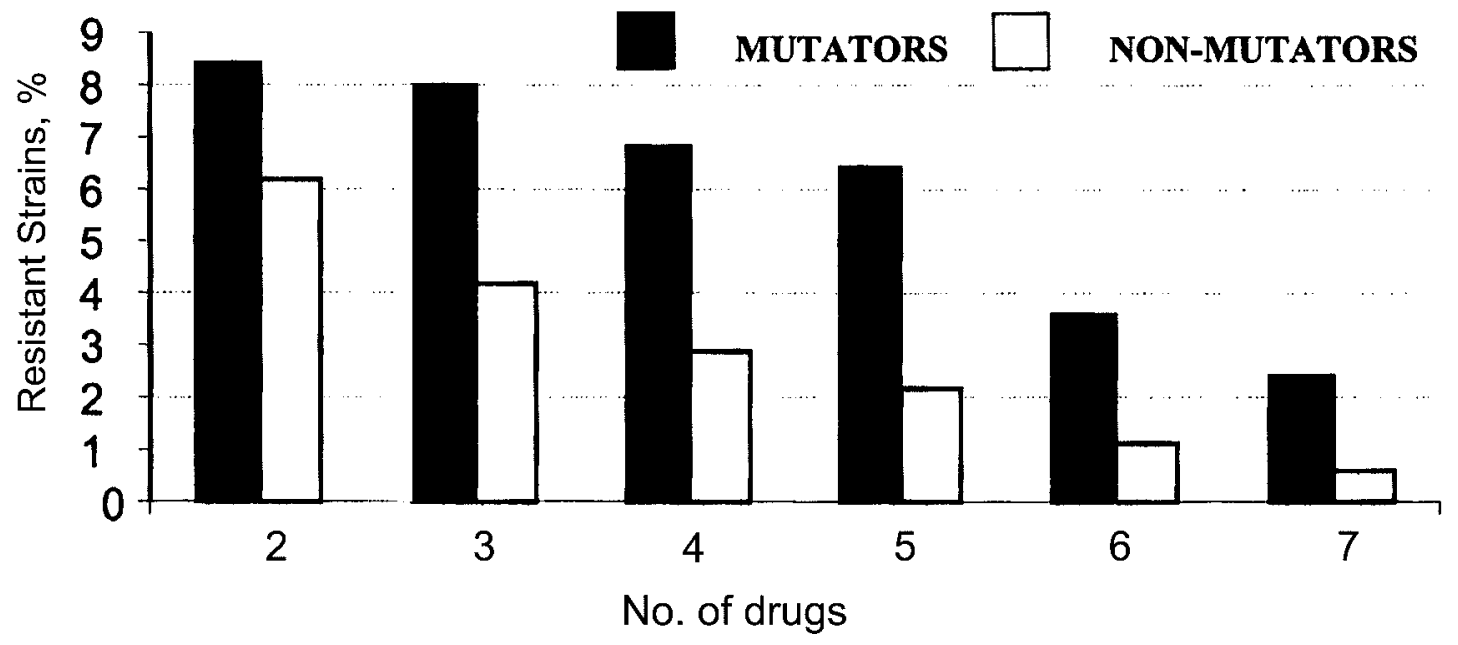

Figure 2. Linkage between hypermutation and multidrug-resistance in $P$. aeruginosa strains isolated from patients with cystic fibrosis. The figure shows the proportions of mutator (black bars) and nonmutator (white bars) strains resistant to different numbers of anti-Pseudomonas drugs. The proportion of multidrug-resistant strains is higher among mutator strains, thus indicating a significant association between hypermutability and multidrugresistance acquisition. 
ever, other investigators have concluded that, despite the fact that a mutS-deficient strain of $S$. aureus had mutation frequencies for antibiotic resistance $\sim 100$-fold greater than those exhibited by the wild type strain, neither stable hypermutation nor transient increases in mutation frequency are likely to play a significant role in the development of antibiotic resistance in S. aureus [69]. Although a direct link between hypermutability in clinical strains and development of vancomycin resistance has to be clearly established, the controversy continues [70, 71]. On the other hand, a conspicuous adaptability to antibiotics has been demonstrated in an E. coli mutator strain by Tanabe et al. [72]. They found that an E. coli mutator strain subjected to ofloxacin pressure followed the mutation history previously observed in quinolone-resistant clinical isolates.

However, despite the amount of data suggesting a relationship between hypermutation and resistance, to date only one study has demonstrated a direct linkage between hypermutable strains and acquisition of antibiotic resistance in pathogens. In this study, Oliver et al. [13] showed that the proportion of strains resistant to 6 different antibiotics was significantly higher among hypermutator $P$. aeruginosa isolates from patients with cystic fibrosis than among nonmutator strains. Additional data from this study indicated that this higher proportion is also found among multidrug-resistant strains; that is, the proportion of $P$. aeruginosa mutator strains harboring multidrug resistance is significantly higher than that of nonmutator strains (figure 2) (A. Oliver and J. B., unpublished data). Altogether, these results suggest that naturally existing mutators may participate in the acquisition of resistance to antibiotics.

The selection of hypermutable strains or the induction of transient hypermutation by antibiotics may explain the unexpected (according to predictions made from laboratory studies), rapid emergence of resistant variants with multiple mutations. For instance, despite the fact that fluoroquinolone resistance in Enterobacteriaceae requires multiple mutations, different genetically-unrelated, fluoroquinolone-resistant E. coli strains have been isolated in different countries [73, 74]. Nevertheless, to demonstrate a clear link between hypermutation and antibiotic resistance in nature, more research is required.

\section{Acknowledgments}

Special thanks are given to Antonio Oliver for his fruitful criticisms and comments on the draft version of the manuscript and for kindly sharing unpublished information. Also, thanks are due to C. Bayliss, J. P. Claverys, L. de Rafael, and J. MartínezBeltrán for their critical review of the manuscript.

\section{References}

1. De la Cruz F, Davies J. Horizontal gene transfer and the origin of species: lessons from bacteria. Trends Microbiol 2000; 8:128-33.
2. Spratt BG. Resistance to antibiotics mediated by target alterations. Science 1994; 264:388-93.

3. Kohler T, Epp SF, Curty LK, et al. Characterization of MexT, the regulator of the MexE-MexF-OprN multidrug efflux system of Pseudomonas aeruginosa. J Bacteriol 1999; 181:6300-5.

4. Poole K. Multidrug efflux pumps and antimicrobial resistance in $P$. aeruginosa and related organisms. J Mol Microbiol Biotechnol 2001; 3: 225-64.

5. Cohen SP, McMurry LM, Hooper DC, et al. Cross-resistance to fluoroquinolones in multiple-antibiotic-resistant (Mar) Escherichia coli selected by tetracycline or chloramphenicol: decreased drug accumulation associated with membrane changes in addition to OmpF reduction. Antimicrob Agents Chemother 1989; 33:1318-25.

6. Livermore, D. M. $\beta$-Lactamases in laboratory and clinical resistance. Clin Microbiol Rev 1995; 8:557-84.

7. Finken M, Kirschner P, Meier A, Wrede A, Bottger EC. Molecular basis of streptomycin resistance in Mycobacterium tuberculosis: alterations of the ribosomal protein S12 gene and point mutations within a functional $16 \mathrm{~S}$ ribosomal RNA pseudoknot. Mol Microbiol 1993; 9:1239-46.

8. Telenti A, Imboden P, Marchesi F, et al. Detection of rifampicinresistance mutations in Mycobacterium tuberculosis. Lancet 1993;341: 647-50.

9. Van Rie A, Warren R, Mshanga I, et al. Analysis for a limited number of gene codons can predict drug resistance of Mycobacterium tuberculosis in a high-incidence community. J Clin Microbiol 2001;39: 636-41.

10. Rasmaswamy S, Musser JM. Molecular genetic basis of antimicrobial agent resistance in Mycobacterium tuberculosis: 1998 update. Tuber Lung Dis 1998; 79:3-29.

11. Blázquez J, Espinosa de los Monteros LE, Samper S, et al. Genetic characterization of multidrug-resistant Mycobacterium bovis strains from a hospital outbreak involving human immunodeficiency viruspositive patients. J Clin Microbiol 1997;35:1390-3.

12. Guerrero A, Cobo J, Fortún J, et al. Nosocomial transmission of $M y$ cobacterium bovis resistant to 11 drugs in people with advanced HIV1 infection. Lancet 1997; 350:1738-42.

13. Oliver A, Cantón R, Campo P, Baquero F, Blázquez J. High frequency of hypermutable Pseudomonas aeruginosa in cystic fibrosis lung infection. Science 2000; 288:1251-3.

14. Blázquez J, Morosini MI, Negri MC, González-Leiza M, Baquero F. Single amino acid replacements at positions altered in naturally occurring extended-spectrum TEM $\beta$-lactamases. Antimicrob Agents Chemother 1995; 39:145-9.

15. Blázquez J, Morosini MI, Negri MC, Baquero F. Selection of naturally occurring extended-spectrum TEM beta-lactamase variants by fluctuating beta-lactam pressure. Antimicrob Agents Chemother 2000; 44 : 2182-4.

16. Medeiros, AA. Evolution and dissemination of $\beta$-lactamases accelerated by generations of $\beta$-lactam antibiotics. Clin Infect Dis 1997; 24 : S19-S45.

17. Blázquez J, Baquero MR, Cantón R, Alós I, Baquero F. Characterization of a new TEM-type $\beta$-lactamase resistant to clavulanate, sulbactam, and tazobactam in a clinical isolate of Escherichia coli. Antimicrob Agents Chemother 1993; 37:2059-63.

18. Luria SE, Delbrück M. Mutations of bacteria from virus sensitivity to virus resistance. Genetics 1943;28:491-511.

19. Newcombe H. Origin of bacterial variants. Nature $1949 ; 164: 150-1$.

20. Lederberg J, Lederberg EM. Replica plating and indirect selection of bacterial mutants. J Bacteriol 1952; 63:399-406.

21. Radman M., Taddei F, Matic I. Evolution-driving genes. Res Microbiol 2000; 151:91-5.

22. Kimura M. On the evolutionary adjustment of spontaneous mutation rates. Genet Res 1967; 9:23-34.

23. Leigh EG. The evolution of mutation rates. Genetics 1973;73:1-18.

24. Drake JW. A constant rate of spontaneous mutation rates in DNAbased microbes. Proc Natl Acad Sci USA 1991; 88:7160-4. 
25. Gibson TC, Scheppe ML, Cox EC. Fitness of an Escherichia coli mutator gene. Science 1970; 169:686-8.

26. Cox EC, Gibson TC. Selection for high mutation rates in chemostats. Genetics 1974; 77:169-84.

27. Chao L, Cox EC. Competition between high and low mutating strains of Escherichia coli. Evolution 1989;37:125.

28. Taddei F, Radman M, Maynard-Smith J, et al. Role of mutator alleles in adaptive evolution. Nature 1997; 387:700-3.

29. Moxon, ER, Rainey PB, Nowak MA, Lenski RE. Adaptive evolution of highly mutable loci in pathogenic bacteria. Curr Biol 1994; 4: 24-33.

30. Leclerc JE, Li B, Payne WL, Cebula TA. High mutation frequencies among Escherichia coli and Salmonella pathogens. Science 1996;274: 1208-11.

31. Matic I, Radman M, Taddei F, et al. Highly variable mutation rates in commensal and pathogenic E. coli. Science 1997; 277:1833-4.

32. Richardson AR, Stojiljkovic I. Mismatch repair and the regulation of phase variation in Neisseria meningitidis. Mol Microbiol 2001;40: 645-55.

33. Prunier AL, Malbruny B, Laurans M, Brouard J, Duhamel JF, Leclercq R. High rate of macrolide resistance in Staphylococcus aureus strains from patients with cystic fibrosis reveals high proportions of hypermutable strains. J Infect Dis 2003; 187:1709-16.

34. Oliver A, Baquero F, Blázquez J. The mismatch repair system (mutS, $m u t L$ and $u v r D$ genes) in Pseudomonas aeruginosa: molecular characterization of naturally occurring mutants. Mol Microbiol 2002; 43: 1641-50.

35. Brégeon D, Matic I, Radman M, Taddei F. J Genet 1999; 78:21-28.

36. Horst JP, Wu T, Marinus MG. Escherichia coli mutator genes. Trends Microbiol 1999; 7:29-36.

37. Modrich P, Lahue R. Mismatch repair in replication fidelity, genetic recombination, and cancer biology. Annu Rev Biochem 1996; 65: 101-33.

38. Matic I, Rayssiguier C, Radman M. Interspecies gene exchange in bacteria: the role of SOS and mismatch repair systems in evolution of species. Cell 1995; 80:507-15.

39. Rayssiguier C, Thaler DS, Radman M. The barrier to recombination between Escherichia coli and Salmonella typhimurium is disrupted in mismatch-repair mutants. Nature 1989;342:396-401.

40. Townsend JP, Nielsen KM, Fisher DS, Hartl DL. Horizontal acquisition of divergent chromosomal DNA in bacteria: effects of mutator phenotypes. Genetics 2003; 164:13-21.

41. Claverys JP, Prudhomme M, Mortier-Barriere I, Martin B. Adaptation to the environment: Streptococcus pneumoniae, a paradigm for recombination-mediated genetic plasticity? Mol Microbiol 2000; 35: 251-9.

42. Dowson CG, Coffey TJ, Spratt BG. Origin and molecular epidemiology of penicillin-binding-mediated resistance to $\beta$-lactam antibiotics. Trends Microbiol 1994; 2:361-6.

43. Claverys JP, Lacks SA. Heteroduplex deoxyribonucleic acid base mismatch repair in bacteria. Microbiol Rev 1986;50:133-65.

44. Humbert O, Parudhomme M, Hakenbeck R, Dowson CG, Claverys JP. Homeologous recombination and mismatch repair during transformation in Streptococcus pneumoniae: saturation of the Hex mismatch repair system. Proc Natl Acad Sci USA 1995; 92:9052-6.

45. Grebe T, Hakenbeck R. Penicillin-binding proteins $2 \mathrm{~b}$ and $2 \mathrm{x}$ of Streptococcus pneumoniae are primary resisance determinants for different classes of beta-lactam antibiotics. Antimicrob Agents Chemother 1996; 40:829-34.

46. Coffey TJ, Daniels M, McDougal LK, Dowson CG, Tenover FC, Spratt BF. Genetic analysis of clinical isolates of Streptococcus pneumoniae with high level resistance to expanded-spectrum cephalosporins. Antimicrob Agents Chemother 1995; 39:1306-13.

47. Kraub J, Hakenbeck R. A mutation in the D,D-carboxypeptidase penicillin-binding protein 3 of Streptococcus pneumoniae contributes to cefotaxime resistance of the laboratory mutant C604. Antimicrob Agents Chemother 1997; 41:936-42.

48. Courcelle J, Khodursky A, Peter B, Brown PO, Hanawalt PC. Comparative gene expression profiles following UV exposure in wild-type and SOS-deficient Escherichia coli. Genetics 2001;158:41-64.

49. Friedberg EC, Wagner R, Radman M. Specialized DNA polymerases, cellular survival, and the genesis of mutations. Science 2002;296: $1627-30$.

50. Sutton MD, Smith BT, Godoy VG, Walker GC. The SOS response: recent insights into $u m u D C$-dependent mutagenesis and DNA damage tolerance. Annu Rev Genet 2000; 34:479-97.

51. Boshoff, HIM, Reed MB, Barry CE III, Mizrahi V. DnaE2 polymerase contributes to in vivo survival and the emergence of drug resistance in Mycobacterium tuberculosis. Cell 2003; 113:183-93.

52. Vulic M, Dionisio F, Taddei F, Radman M. Molecular keys to speciation: DNA polymorphism and the control of genetic exchange in enterobacteria. Proc Natl Acad Sci USA 1997; 94:9763-7.

53. Matic I, Taddei F, Radman M. No genetic barriers between Salmonella enterica serovar Typhimurium and Escherichia coli in SOS-induced mismatch repair-deficent cells. J Bacteriol 2000; 182:5922-4.

54. Li B, Tsui HCT, LeClerc JE, et al. Molecular analysis of $m u t S$ expression and mutation in natural isolates of pathogenic Escherichia coli. Microbiology 2003; 149:1323-31.

55. Bjedov I, Tenaillon O, Gerard B, et al. Stress-induced mutagenesis in bacteria. Science 2003; 300:1404-9.

56. Karunakaran P, Davies J. Genetic antagonism and hypermutability in Mycobacterium smegmatis. J Bacteriol 2000; 182:3331-5.

57. Björkman J, Nagaev I, Berg OG, Hughes D, Andersson DI. Effects of environment on compensatory mutations to ameliorate costs of antibiotic resistance. Science 2000;287:1479-82.

58. Böttger EC, Springer B, Pletschette M, Sander P. Fitness of antibioticresistant microorganisms and compensatory mutations. Nat Med 1998; 4:1343-4.

59. Mao EF, Lane L, Lee J, Miller JH. Proliferation of mutators in a cell population. J Bacteriol 1997; 179:417-22.

60. Giraud A, Matic I, Radman M, Fons M, Taddei F. Mutator bacteria as a risk factor in treatment of infectious diseases. Antimicrob Agents Chemother 2002; 46:863-5.

61. Gilligan PH. Microbiology of cystic fibrosis lung disease. In: Yankaskas and Knowles, eds. Cystic fibrosis in adults. Philadelphia: LippincottRaven, 1999:93-114.

62. Rosche WA, Foster P. Mutation under stress: adaptive mutation in Escherichia coli. In: Bacterial stress responses. Storz G and HenggeAronis R, eds. Washington DC: ASM Press, 2000.

63. Phillips I, Culebras E, Moreno F, Baquero F. Induction of the SOS response by new 4-quinolones. J Antimicrob Chemother 1987;20: 631-8.

64. Piddock LJ, Wise R. Induction of the SOS response in Escherichia coli by 4-quinolone antimicrobial agents. FEMS Microbiol Lett 1987; 41: 289-94.

65. Ysern P, Clerch B, Castaño M, Gilbert I, Barbé J, Llagostera M. Induction of SOS genes in Escherichia coli and mutagenesis in Salmonella typhimurium by fluoroquinolones. Mutagenesis 1990;5:63-6.

66. Ren L, Rahman MS, Humayun MZ.Escherichia coli cells exposed to streptomycin display a mutator phenotype. J Bacteriol 1999;181: 1043-4.

67. Baquero MR, Gómez-Gómez JM, Lobo M, Baquero F, Blázquez J. $\beta$ lactams induce $\operatorname{din} B$ (error-prone DNA-polymerase IV) transcription in Escherichia coli, resulting in a mutator phenotype [abstract 1804]. In: Programs and abstracts of the 41st Interscience Conference on Antimicrobial Agents and Chemotherapy (Chicago). Washington DC: American Society for Microbiolgoy, 2001:99.

68. Schaaff F, Reipert A, Bierbaum G. An elevated mutation frequency favors development of vancomycin resistance in Staphylococcus aureus. Antimicrob Agents Chemother 2002; 46:3540-8.

69. O'Neill AJ, Chopra I. Insertional inactivation of mutS in Staphylococcus 
aureus reveals potential for elevated mutation frequencies, although the prevalence of mutators in clinical isolates is low. J Antimicrob Chemother 2002; 50:161-9.

70. O’Neill AJ, Chopra I. Lack of evidence for involvement of hypermutability in emergence of vancomycin-intermediate Staphylococcus aureus. Antimicrob Agents Chemother 2003; 47:1484-5.

71. Bierbaum G, Reipert A, and Schaaff F. Authors reply. Antimicrob Agents Chemother 2003; 47:1485.

72. Tanabe K., Kondo T, Onodera Y, Furusawa M. A conspicuous adapt- ability to antibiotics in the Escherichia coli mutator strain, dnaQ49. FEMS Microbiol Lett 1999; 176:191-6.

73. Garau J, Xercavins M, Rodriguez-Carballeira M, et al. Emergence and dissemination of quinolone-resistant Escherichia coli in the community. Antimicrob Agents Chemother 1999; 43:2736-41.

74. Wang H, Dzink-Fox JL, Chen M, Levy SB. Genetic characterization of highly fluoroquinolone-resistant clinical Escherichia coli strains from China: role of acrR mutations. Antimicrob Agents Chemother 2001; 45:1515-21. 